\title{
Optimization of Propagation in Interval Constraint Networks for Tolerance Design
}

\author{
Frank W. Ciarallo" and Christopher C. Yang ${ }^{\dagger}$ \\ *: Department of Systems and Industrial Engineering \\ The University of Arizona \\ $\dagger$ : Department of Computer Science \\ The University of Hong Kong \\ Email: yang@cs.hku.hk
}

\begin{abstract}
This paper proposes a hierarchical interval constraint network and interval propagation techniques for automatic tolerance design. The nodes in interval constraint networks represent the entities, the attributes, and the functional requirements of the mechanical design or the constraint functions. The arcs represent the relationships between the entities, the attributes, the functional requirements and the constraint functions. We developed the forward propagation technique for tolerance analysis and the backward propagation technique for tolerance synthesis. In tolerance analysis, given the entity tolerances, the goal is to ensure that the functional requirement tolerances are met. In tolerance synthesis, given the functional requirement tolerances, the goal is to synthesize a new set of entity tolerances. In backward propagation, the minimization of the manufacturing cost is also considered. During backward propagation, the tolerances of entities, which have a smaller impact on manufacturing costs, will be tightened first. Using this mechanism, we ensure the constraints are satisfied and the manufacturing costs are minimized.
\end{abstract}

\section{Introduction}

Tolerance design plays an important role in the relationship between performance and the manufacturing costs of a product. Decreasing the tolerance range will improve performance but will also increase the manufacturing costs. It is desirable to optimize the manufacturing costs under the constraints of product design, the relationships between tolerances of the entities' dimensions and tolerances of the functional requirements. In this paper, we develop a constraintbased reasoning mechanism to analyze a given set of design tolerances and synthesize a new set of tolerances to satisfy the functional requirements of a product. We have also considered the minimization of manufacturing cost during the tolerance propagation in interval constraint networks.

\section{List of Symbols}

V variable (corresponding to entity, attribute, and functional requirement)

$\mathrm{V}$ assigned for $\mathrm{V}$

$v_{u p} \quad$ upper limit of interval $\mathrm{V}$

Vlow lower limit of $\mathrm{V}$

$v_{\text {nom }}$ nominal value of the variable

$\mathrm{v}$ any value in $\mathrm{V}$
For a given design of a mechanical part, a relationship can be derived for the functional requirement in terms of the entities. This relationship can be expressed as: $\mathbf{Y}=\mathbf{f}\left(\mathbf{X}_{1}, \mathbf{X}_{2}, \ldots, \mathbf{X}_{\mathbf{n}}\right)$ where $\mathbf{Y}$ is the functional requirement and $\mathbf{X}_{\mathbf{i}}$ is the $i^{\text {th }}$ entity. $\mathbf{n}$ is the number of entities that are related by the equation to the corresponding functional requirement.

In tolerance analysis, the entity tolerances, $\mathrm{X}_{1}, \mathrm{X}_{2}$, $\ldots, X_{n}$, are given. The goal is to ensure that the functional requirement tolerance, $Y$, is met. The tolerances $\mathrm{X}_{\mathrm{i}}$ and $\mathrm{Y}$, are the range of acceptable values for, $\mathbf{X}_{\mathbf{i}}$ and $\mathbf{Y}$, respectively. If the assigned functional requirement tolerances are not met, the tolerances for the entities need to be reassigned by tolerance synthesis in order to achieve the functional requirements.

In tolerance synthesis, the functional requirement tolerance, $\mathrm{Y}$, is given. The goal is to determine a set of feasible entity tolerances, $X_{1}, X_{2}, \ldots, X_{n}$, to fulfill the functional requirement. The task of tolerance synthesis is more difficult because $n$ entity tolerances are determined based on one functional requirement tolerance. In contrast, in tolerance analysis, one functional requirement tolerance is determined based on $\mathbf{n}$ entity tolerances. Figure 1 gives the concept and relationship of tolerance analysis and synthesis.

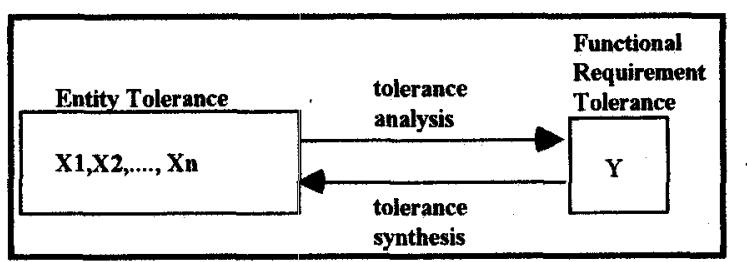

Figure 1. Relationship of tolerance synthesis and tolerance analysis.

\subsection{Related Works in Tolerance Design}

The previous works on tolerance design are summarized as below. Foster [6] developed techniques for tolerance calculation. Turner et al. [19] presented a worst-case tolerance analysis. Fortini [5] and Parkinson [15] applied statistical analysis techniques. Michael and Siddall [12], and Cagan et al. [1] have applied optimization techniques for tolerance synthesis. These techniques include linear and nonlinear programming, and simulated annealing. Lu and Wilhelm [10] proposed a tolerance synthesis approach, CASCADE-T that used a 
representation of the conditional tolerance relations that exist between features of a part.

\subsection{Related Work in Interval Constraints}

Interval Constraint satisfaction problems (ICSPs) are often formulated in AI tasks. A constraint network is a declarative structure that consists of nodes and arcs. The nodes represent the variables or the constraints. The arcs represent the relationship between the variables and the constraints. The variables are labeled by intervals, or sets of possible values. The constraints include any type of mathematical operation or binary relation. Constraint propagation is utilized to perform inferences about quantities. For different types of variables and definitions of satisfaction in constraint satisfaction problems, different propagation techniques can be formulated. For tolerance design, the variables are labeled by intervals and the constraints are n-ary mathematical operations.

Dechter and Pearl [4], Mackworth and Freduer [11] has considered interval constraint networks with the domains of the variables as discrete, finite sets. Ladkin and Reinefeld [9] has studied the constraints with binary relations. Davis and Hyvonen's work is most closely related to ours. The constraints in their interval constraint satisfaction problems (ICSPs) are n-ary mathematical operations and the intervals are real intervals. While these methods are able to determine the solutions for ICSPs, their definition of consistency and satisfaction of the constraint network is not appropriate in the problem of tolerance synthesis and analysis of mechanical parts. Therefore, these techniques cannot solve our problem. The differences of the definitions and applications between their work and our work are presented in $[20,21]$.

\subsection{Contribútions}

We have approached the problem of tolerance design by combining interval constraint network and interval propagation techniques. The contributions of our work can be summarized as follows: (i) developing hierarchical interval constraint network to represent the relationships between the entities, attributes, and functional requirement of a mechanical part, (ii) develop forward and backward propagation techniques, and (iii) optimizing manufacturing costs in backward propagation.

\section{Interval Constraint Network for Tolerance Design}

\subsection{Hierarchical Interval Constraint Network and} Constraint Functions

For each mechanical design, the relationship between the highest level, functional requirement, and the lowest level, entity, can be represented by a hierarchical network. The functional requirement describes the functions of the design and the requirement to satisfy these functions. Each functional requirement can be described as a function (constraint) in terms of attributes. For example, the functional requirement, volume of $a$ sphere, can be described as a function of the attribute, inner radius. An attribute is also described as a function in terms of the mechanical part's entities. The inner radius can be computed as a function in terms of the outer radius and the thickness of the material. These relationships are described as a hierarchical interval constraint network as shown in Figure 2 in our approach.

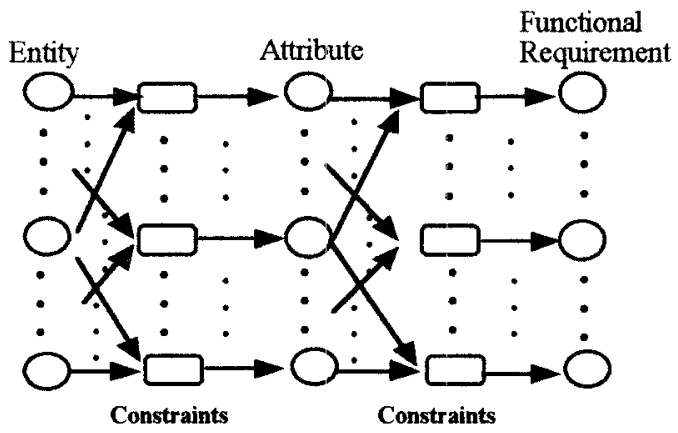

Figure 2. A hierarchical interval constraint network representing the constraints between the entities, attributes, and functional requirements of the mechanical design.

\subsection{Satisfaction of Interval Constraint Networks for Tolerance Design}

The definition of satisfaction in the interval constraint network always depends on the purpose of application. A good understanding of the goal of constraint satisfaction for the problem investigated and an appropriate definition of the satisfaction of the constraint network are the foundations of a successful constraint network solution to the problem.

In ICSP, according to Hyvonen [7], the satisfaction of the interval constraint network is defined as follows:

- A variable, $\mathbf{V}_{\mathbf{i}}$, is consistent if and only if

$$
\begin{aligned}
& \forall\left(\mathrm{v}_{\mathrm{i}} \in \mathrm{V}_{\mathrm{i}} \mid \mathrm{V}_{\mathrm{i}}=\mathrm{v}_{\mathrm{i}}\right), \\
& \exists\left(\mathrm{v}_{1} \in \mathrm{V}_{1}, \ldots, \mathrm{v}_{\mathrm{i}-1} \in \mathrm{V}_{\mathrm{i}-1}, \mathrm{v}_{\mathrm{i}+1} \in \mathrm{V}_{\mathrm{i}+1}, \ldots, \mathrm{v}_{\mathrm{n}} \in \mathrm{V}_{\mathrm{n}} \mid\right. \\
& \left.\mathbf{V}_{1}=\mathrm{v}_{1}, \ldots, \mathrm{V}_{\mathrm{i}-1}=\mathrm{v}_{\mathrm{i}-1}, \mathrm{~V}_{\mathrm{i}+1}=\mathrm{v}_{\mathrm{i}+1}, \ldots, \mathrm{V}_{\mathrm{n}}=\mathrm{v}_{\mathrm{n}}\right)
\end{aligned}
$$

such that all constraints are satisfied.

- The constraint network is satisfied if and only if all variables are consistent

The properties of the consistency of a variable as described in ICSP are not appropriate for tolerance design. For example, $A=f(L, W)=\mathbf{L} \cdot \mathbf{W}$, where $A$ is area, $L$ is length, and $W$ is width of a rectangle. Given the tolerances, $\mathrm{L}, \mathrm{W}$, and $\mathrm{A}, \mathrm{F}(\mathrm{L}, \mathrm{W})$ must be a subset of A so that the designed tolerances for length and width satisfy the expected tolerance of the rectangular. $(F O)$ is interval constraint function based on $\mathrm{f}(\mathrm{)}$.)However, using this definition, even though all the variables in the network are consistent, the usual definition of satisfying a mechanical tolerance is not satisfied.

Since the purpose of tolerance design in an interval constraint network is different from the purpose of ICSP, a new definition of satisfaction is required. The 
satisfaction of the network depends on the consistency of the components in the network. In ICSP, the satisfaction of the network is defined in terms of the consistency of the variables. However, based on the application of tolerance design, the definition of consistency should focus on constraints.

In a constraint network for tolerance design, the constraint is multiple/single inputs and single output (MISO or SISO) and is represented as a triple, $\mathrm{C}_{\mathrm{i}}(\mathrm{U}, \mathrm{k}, \mathrm{f}(\mathrm{)})$. $\mathrm{U}$ is the set of indexes for the input variables and $\mathrm{k}$ is the index of the output variable for the constraint $\mathrm{C}_{\mathrm{i}}$. $\mathrm{fO}$ is the constraint function. For example, $\mathrm{U}=\{1,2\}$ and $\mathbf{k}=3$, if the constraint function of $\mathrm{C}_{1}$ is $V_{3}=$ $\mathrm{f}\left(\mathbf{V}_{\mathbf{1}}, \mathbf{V}_{\mathbf{2}}\right)$

The definitions of consistency of a constraint and satisfaction of the interval constraint network for tolerance design are as follows:

\section{Definition 1:}

A constraint, $\mathrm{C}_{\mathrm{i}}(\mathrm{U}, \mathrm{k}, \mathrm{f}(\mathrm{)})$, is consistent if and only if

$\frown\left(\forall v_{j} \in V_{j} \mid V_{j}=v_{j}\right),\left(\exists v_{k} \in V_{k} \mid V_{k}=v_{k}\right)$

such that $\mathrm{C}_{\mathrm{i}}(\mathrm{U}, \mathrm{k})$ is satisfied.

where $\mathrm{U}$ is the set of indexes for the input variables and $\mathrm{k}$ is the index of the output variable for the constraint Ci.

\section{Definition 2:}

The interval constraint network for tolerance design is satisfied if and only if all of the constraints are consistent.

Based on the definitions of constraint consistency and network satisfaction, the tolerances assigned to the entities are ensured to satisfy the tolerances of the functional requirements. The interval computed from the input intervals and the interval constraint function is expected to be a subset of the assigned output interval for each constraint in the network.

\section{Tolerance Propagation}

With these new definitions of consistency and satisfaction for tolerance design, new tolerance propagation techniques are needed. With such techniques, tolerances can be propagated from variable to variable in the constraint network to ensure that the constraints are consistent.

Tolerance propagation is utilized to update the intervals in the network to make the interval constraints consistent. Tolerance can be propagated from the input intervals of a constraint to the single output interval, which is known as forward propagation. Tolerance can also be propagated from the single output interval of a constraint to multiple input intervals, known as backward propagation. The forward and backward propagation techniques for tolerance design are developed based on Definitions 1 and 2. Given a constraint with constraint function $\mathbf{X}_{\mathbf{k}}=\mathbf{f}\left(\mathbf{X}_{1}, \mathbf{X}_{\mathbf{2}}, \ldots, \mathbf{X}_{\mathbf{n}}\right)$, with input intervals, $\mathrm{X}_{1}, \mathrm{X}_{2}, \ldots, \mathrm{X}_{\mathrm{n}}$, and output interval, $\mathrm{X}_{\mathrm{k}}$, if the constraint is not consistent $\left(F\left(X_{1}, X_{2}, \ldots, X_{n}\right)\right.$ is not a subset of $\left.X_{k}\right)$, either $\mathrm{X}_{\mathrm{k}}$ must be relaxed (widened) or one or more of the input intervals must be tightened (narrowed). $X_{k}$ is relaxed by propagating $X_{1}, X_{2}, \ldots$, and $X_{n}$ forward. $X_{1}, X_{2}, \ldots$, and $X_{n}$ are tightened by propagating $X_{k}$ backward

\subsection{Forward Propagation for a Single Constraint}

The forward propagation is based on the constraint function such that the intervals of the input variables are propagated to the interval of the single output variable. If the interval propagated from the input intervals is not a subset of the output interval, the output interval is updated (relaxed) to the union of the propagated interval and the original assigned output interval, otherwise, the constraint is consistent and nothing is changed. The algorithm for forward propagation is given as:

Forward Propagation for constraint, $\mathrm{C}(\{1,2, \ldots, \mathbf{n}\}, \mathbf{k}, \mathrm{f}())$, $\mathrm{FP}\left(\mathbf{X}_{\mathbf{1}}, \mathbf{X}_{\mathbf{2}}, \ldots, \mathbf{X}_{\mathbf{n}} ; \mathbf{X}_{\mathbf{k}}\right)$

Propagated from Input Tolerance to the Upper Limit of the Output Tolerance

$$
\mathrm{x}_{\mathrm{kpp}_{\mathrm{up}}}=\mathrm{f}\left(\mathrm{x}_{\mathbf{1} \varphi}, \ldots, \mathrm{x}_{\mathrm{n} \varphi}\right)
$$

where $\mathbf{x}_{\mathbf{i} \varphi}=\mathrm{x}_{\mathrm{i}_{\mathrm{up}}}$ if $\mathbf{X}_{\mathbf{k}}$ is monotonic increasing with respect to $\mathbf{X}_{\mathbf{i}}$.

$$
\begin{aligned}
& \mathrm{x}_{\mathbf{i} \varphi}=\mathrm{x}_{\mathbf{l}_{\text {low }}} \text { if } \mathbf{X}_{\mathbf{k}} \text { is monotonic decreasing with } \\
& \text { respect to } \mathbf{X}_{\mathbf{i}} \text {. }
\end{aligned}
$$

Propagated from Input Tolerance to the Lower Limit of the Output Tolerance

$$
\mathrm{x}_{\mathrm{k}_{\text {low }}}{ }^{\prime}=\mathrm{f}\left(\mathrm{x}_{1 \mathrm{~K}}, \ldots, \mathrm{x}_{\mathrm{nK}}\right)
$$

where $x_{i k}=x_{i_{u p}}$ if $\mathbf{X}_{\mathbf{k}}$ is monotonic increasing with respect to $\mathbf{X}_{\mathbf{i}}$.

$\mathrm{x}_{\mathbf{i k}}=\mathrm{x}_{\mathbf{i} \text { low }} \quad$ if $\mathbf{X}_{\mathbf{k}}$ is monotonic decreasing with respect to $\mathbf{X}_{\mathbf{i}}$.

Relaxing the Output Tolerance

$$
\begin{aligned}
& \text { If } \mathbf{x}_{\mathbf{k}_{\mathbf{u p}}}{ }<\mathbf{x}_{\mathbf{k}_{\text {low }}} \text { or } \mathbf{x}_{\mathbf{k}_{\text {low }}}{ }^{\prime}>\mathrm{x}_{\mathbf{k}_{\mathbf{u p}}} \text {, } \\
& \text { NO SOLUTION } \\
& \mathbf{x}_{\mathrm{k}_{\mathrm{up}}}=\mathrm{x}_{\mathrm{k}_{\mathrm{up}}} \quad \text { if } \mathrm{x}_{\mathrm{k}_{\mathrm{up}}}{ }^{\prime}>\mathrm{x}_{\mathrm{k}_{\mathrm{up}}} \\
& \mathrm{x}_{\mathbf{k}_{\text {low }}}=\mathrm{x}_{\mathrm{k}_{\text {low }}}{ }^{\prime} \quad \text { if } \mathrm{x}_{\mathrm{k}_{\text {low }}}{ }^{\prime}<\mathrm{x}_{\mathrm{k}} \text { low }
\end{aligned}
$$

\subsection{Backward Propagation for a Single Constraint}

The backward propagation is also based on the constraint function such that the interval of the output variable is propagated to one or more of the intervals of the input variables. If the constraint is not consistent, the output interval is propagated to the input intervals by tightening each of the input intervals. We integrate the manufacturing cost functions with the backward propagation in order to minimize the increase of cost when we tighten the tolerances. 
3.2.1 Manufacturing Cost Functions and Sensitivity

Several researchers $[8,16,17,18]$ have modeled the relationship between tolerance (accuracy of manufactured dimensions) and manufacturing cost of achieving that accuracy. For examples, Spotts [18] developed a model based on the inverse square of tolerance; Speckhart [17] developed an exponential model. In this paper, we utilize the exponential model, which is the most commonly used.

Manufacturing Cost Function for the tolerance upper limit:

$$
\mathrm{MC}_{\mathbf{X} \boldsymbol{} \varphi}(\mathrm{x})=\mathrm{A}_{\varphi}+\mathrm{B}_{\varphi} \operatorname{Exp}\left(\mathrm{C}_{\varphi}\left(\mathrm{x}-\mathrm{x}_{\mathrm{inom}_{\mathrm{n}}}\right)\right)
$$

Manufacturing Cost Function for the tolerance lower limit:

$$
\mathrm{MC}_{\mathrm{xiK}_{\mathrm{K}}}(\mathrm{x})=\mathrm{A}_{\mathrm{K}}+\mathrm{B}_{\mathrm{K}} \operatorname{Exp}\left(\mathrm{C}_{\mathrm{K}}\left(\mathrm{x}_{\mathrm{inom}_{\text {nom }}}-\mathrm{x}\right)\right)
$$

Figure 3 shows examples of the manufacturing cost functions of the upper and lower limits.

\section{Manufacturing Cost}

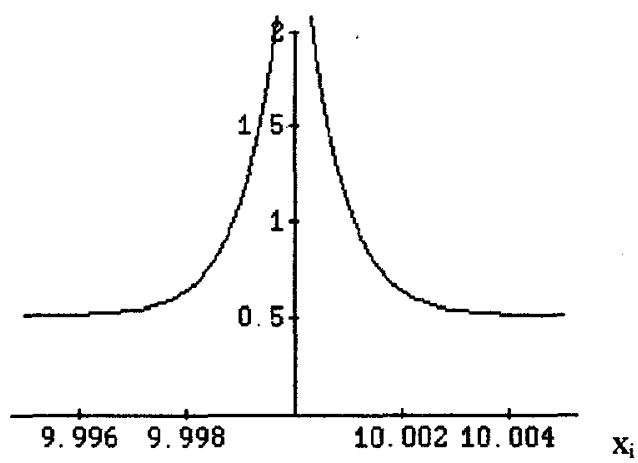

Figure 3 Manufacturing cost functions of the upper limit and lower limits, where $x_{i n o m}=10.0, A_{\varphi}=A_{K}=$ $0.515, \mathrm{~B}_{\varphi}=\mathrm{B}_{\mathrm{K}}=2.52, \mathrm{C}_{\varphi}=\mathrm{C}_{\mathrm{K}}=-1473.0$.

The sensitivity of tolerance is the ratio of the change in manufacturing cost to the change in dimension. It is formulated as follow:

Sensitivity of $\mathrm{xi \varphi}$ :

$$
\begin{aligned}
\mathrm{S}\left(\mathrm{x}_{\mathrm{i}_{\varphi}}\right) & =\left.\frac{\delta \mathrm{MC}_{\mathrm{x}_{\text {ip }}}(\mathrm{x})}{\delta \mathrm{x}}\right|_{\mathrm{X}=\mathrm{x}_{\mathrm{i}_{\varphi}}} \\
& =\mathrm{B}_{\varphi} \mathrm{C}_{\varphi} \operatorname{Exp}\left(\mathrm{C}_{\varphi}\left(\mathrm{x}_{\mathrm{i} \varphi}-\mathrm{x}_{\mathrm{i}_{\text {nom }}}\right)\right)
\end{aligned}
$$

Sensitivity of $x_{i k}$ :

$$
\begin{aligned}
\mathrm{S}\left(\mathrm{x}_{\mathrm{i} K}\right) & =\left.\frac{\delta \mathrm{MC}_{\mathrm{x}_{\mathrm{i \kappa}}}(\mathrm{x})}{\delta \mathrm{x}}\right|_{\mathrm{X}=\mathrm{x}_{\mathrm{i}_{\boldsymbol{\kappa}}}} \\
& =\mathrm{B}_{\mathrm{K}} \mathrm{C}_{\mathrm{K}} \operatorname{Exp}\left(\mathrm{C}_{\mathrm{K}}\left(\mathrm{x}_{\mathrm{i}_{\text {nom }}}-\mathrm{x}_{\mathrm{iK}}\right)\right)
\end{aligned}
$$

Figure 4 shows examples of the sensitivity of the upper and lower limits.

Sensitivity

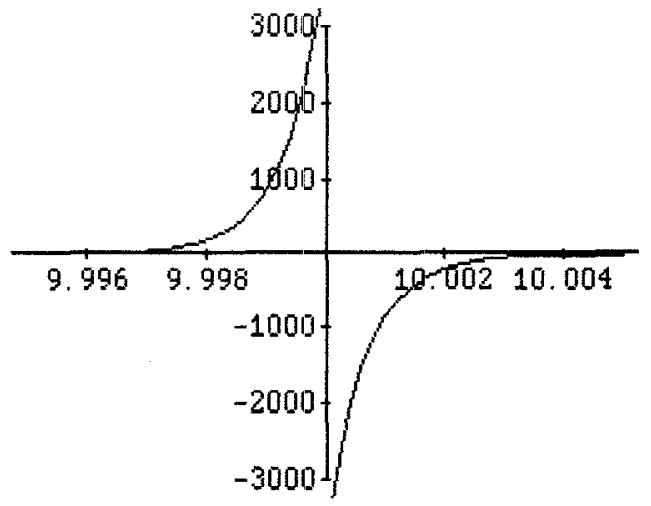

Figure 4 Sensitivity of the upper limit and lower limit using the manufacturing cost functions in Figure 3.

\subsubsection{Optimization}

To integrate the optimization of manufacturing cost with the backward propagation, the optimization problem is formulated as follow:

Minimize $\sum_{i=1}^{n} M_{x_{i \varphi}}\left(x_{i_{\varphi}}-\Delta x_{i_{\phi}}\right)$

Subject to

$$
\mathrm{f}\left(\mathrm{x}_{1_{\varphi}}-\Delta \mathrm{x}_{1_{q}}, \mathrm{x}_{2_{\varphi}}-\Delta \mathrm{x}_{2_{\varphi}}, \ldots, \mathrm{x}_{\mathrm{n}_{\varphi}}-\Delta \mathrm{x}_{\mathrm{n}_{\varphi}}\right) \leq \mathrm{x}_{\mathrm{r}_{\mathrm{up}}}
$$

$\Delta \mathbf{x}_{i_{\varphi}} \geq 0 \quad$ if $\mathbf{X}_{\mathbf{k}}$ is monotonic increasing with respect to $\mathbf{X}_{\mathbf{i}}$ $\Delta x_{i_{q}} \leq 0$ if $\mathbf{X}_{k}$ is monotonic decreasing with respect to $X_{i}$ for $1 \leq i \leq n$

Minimize $\sum_{\mathbf{i}=1}^{\mathrm{n}} \mathrm{MC}_{\mathbf{x}_{\mathrm{i} \kappa}}\left(\mathbf{x}_{\mathbf{i}_{\kappa}}+\Delta \mathrm{x}_{\mathrm{i}_{\kappa}}\right)$

Subject to

$\mathrm{f}\left(\mathrm{x}_{1_{\kappa}}-\Delta \mathrm{x}_{1_{k}}, \mathrm{x}_{2_{\kappa}}-\Delta \mathrm{x}_{2_{\kappa}}, \ldots, \mathrm{x}_{\mathbf{n}_{\kappa}}-\Delta \mathrm{x}_{\mathbf{n}_{\kappa}}\right) \geq \mathrm{x}_{\mathbf{1}_{\text {low }}}$

$\Delta \mathbf{x}_{i_{q}} \leq 0$ if $\mathbf{X}_{\mathbf{k}}$ is monotonic increasing with respect to $\mathbf{X}_{\mathbf{i}}$

$\Delta x_{i_{p}} \geq 0$ if $\mathbf{X}_{\mathbf{k}}$ is monotonic decreasing with respect to $X_{i}$ for $1 \leq \mathrm{i} \leq \mathrm{n}$

where $\mathrm{MC}_{\mathrm{Xi} \varphi}(\mathrm{x})$ and $\mathrm{MC}_{\mathrm{X}_{\mathbf{i}}}(\mathrm{x})$ are the manufacturing cost of $x_{i \varphi}$ and $x_{i \kappa}$, respectively. 
Backward Propagation with Optimization $X_{\text {iq }}$ :

1. Compute $\left|\mathrm{S}\left(\mathrm{x}_{\mathrm{i}} \varphi\right)\right|$, arrange them in ascending order and save the corresponding index in a set Sen.

2. Initialize $\mathbf{k}$ to 1 .

3. Retrieve the first $k$ element of Sen.

Change all the corresponding $\mathrm{x}_{\mathrm{i} \varphi}$ by $\Delta \mathrm{x}_{\mathrm{i} \varphi}$ such that all their corresponding $\left|\mathrm{S}\left(\mathrm{x}_{\mathrm{i} \varphi}\right)\right|$ are the same.

4. If

$$
f\left(x_{1_{\varphi}}-\Delta x_{1_{\varphi}}, x_{2_{\varphi}}-\Delta x_{2_{\varphi}}, \ldots, x_{n_{\varphi}}-\Delta x_{n_{\varphi}}\right)>x_{k_{u p}}
$$

If the sensitivities of the first $k$ elements are smaller than that of the next element of Sen. Repeat 3.

Else

$$
\begin{aligned}
& \mathbf{k}=\mathbf{k}+\mathbf{1} \\
& \text { If } \mathbf{k}>\mathbf{n}, \text { NO SOLUTION. } \\
& \text { Repeat } 3
\end{aligned}
$$

Else

Save the current values of $\mathrm{x}_{i \varphi}$ as solution.

Backward propagation with optimization from $\mathrm{X}_{\mathrm{ilow}}$ is developed similarly.

\section{Example:}

Figure 5 shows an example of shaft and bearing. The constraint function is as follow:

$$
c=\mathbf{d}_{\mathbf{0}}-\mathbf{d i}_{\mathbf{i}}
$$

where $\mathbf{c}$ is the clearance, $\mathbf{d}_{0}$ and $\mathbf{d}_{\mathbf{i}}$ are the diameters.

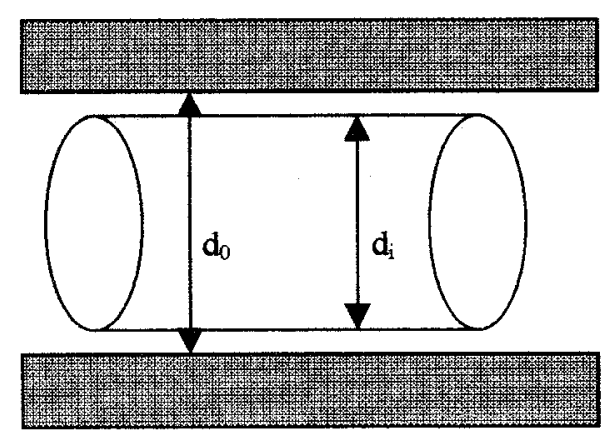

Figure 5 Shaft of Bearing

The upper limit of clearance is in terms of the lower limit of $\mathrm{d}_{0}$ and upper limit of $\mathrm{d}_{\mathrm{i}}$, and the lower limit of clearance is in terms of the upper limit of $d_{0}$ and lower limit of $\mathrm{d}$ :

$$
\begin{aligned}
& c_{u p}=d_{0}{ }_{u p}-d_{\text {ilow }} \\
& c_{\text {low }}=d_{0 l o w}-d_{\text {iup }}
\end{aligned}
$$

Optimization problem in Backward Propagation:

Minimize

$$
\begin{gathered}
M C_{d 0}\left(d_{0} 0_{\text {up }}-\Delta d_{0}\right)+ \\
M C_{d_{i}}\left(d_{\text {ilow }}-\Delta d_{\text {ilow }}\right)
\end{gathered}
$$

Subject to

$$
\begin{aligned}
& \left(\mathrm{d}_{0_{\text {up }}}-\Delta \mathrm{d}_{0_{\text {up }}}\right)-\left(\mathrm{d}_{\mathrm{i}_{\text {low }}}-\Delta \mathrm{d}_{\mathrm{i}_{\text {low }}}\right) \leq \mathrm{c}_{\mathrm{up}} \\
& \Delta \mathrm{d}_{0_{\text {up }}} \geq 0 \\
& \Delta \mathrm{d}_{\mathrm{i}_{\text {low }}} \leq 0
\end{aligned}
$$

Initial settings:

$$
\begin{aligned}
& \mathrm{d}_{0_{\text {up }}}=10.005, \mathrm{~d}_{\text {ilow }}=9.996, \mathrm{c}_{\text {up }}=0.007, \\
& \mathrm{~d}_{0_{\text {nom }}}=10.003, \mathrm{~d}_{\text {inom }_{\text {nom }}}=9.998, \mathrm{c}_{\text {nom }}=0.005
\end{aligned}
$$

Manufacturing cost functions of diameters:

$$
\begin{aligned}
& M C_{\mathrm{d} 0}(\mathrm{x})=0.515+2.52 \operatorname{Exp}\left(-1473\left(\mathrm{x}-\mathrm{d}_{0} 0_{\text {nom }}\right)\right) \\
& \mathrm{MC}_{\mathrm{di}_{\mathrm{i}}}(\mathrm{x})=1.11+3.11 \operatorname{Exp}\left(-1132\left(\mathrm{~d}_{\text {inom }_{\text {nom }}}-\mathrm{x}\right)\right)
\end{aligned}
$$

Backward Propagation:

1. $\left|\mathrm{S}\left(\mathrm{d}_{0} \mathrm{up}\right)\right|=195.06$

$\left|\mathrm{S}\left(\mathrm{d}_{\text {ilow }}\right)\right|=365.90$

$\operatorname{Sen}=\left\{\mathrm{d}_{0 \text { up }}, \mathrm{d}_{\mathrm{ilow}}\right\}$

2. $\mathrm{k}=1$

3. $\Delta \mathrm{d}_{0 \mathrm{up}}=0.0004271$,

$\mathrm{d}_{0}$ up $=10.0045729$

4. $\mathrm{d}_{0_{\text {up }}}-\mathrm{d}_{\text {ilow }}=0.0085729>0.007=\mathrm{c}_{\text {up }}$

$\mid \mathrm{S}\left(\mathrm{d}_{0}\right.$ up $\left._{\text {p }}\right)|=365.92>| \mathrm{S}\left(\mathrm{d}_{\text {ilow }}\right) \mid=365.90$

$$
\mathrm{k}=2=\mathrm{n}
$$

3. $\Delta \mathrm{d}_{0 \mathrm{up}}=0.0006834$,

$\mathrm{d}_{0 \text { up }}=10.00389$,

$\Delta \mathrm{d}_{\text {ilow }}=-0.00089$,

$\mathrm{d}_{\mathrm{ilow}}=9.99689$,

$\left|\mathrm{S}\left(\mathrm{d}_{0_{\mathrm{up}}}\right)\right|=1000.59$,

$\left|\mathrm{S}\left(\mathrm{d}_{\mathrm{ilow}}\right)\right|=1002.09$,

4. $\quad \mathrm{d}_{0 \text { up }}-\mathrm{d}_{\text {ilow }}=0.007=\mathrm{c}_{\text {up }}$

Therefore, the solution is $\mathrm{d} 0_{\mathrm{up}}=10.00389$, and $\mathrm{d}_{\text {ilow }}=9.99689, \mathrm{~d}_{0}$ up and $\mathrm{d}_{\text {ilow }}$ can be solved similarly by propagating $c_{\text {low }}$ backward.

\section{Conclusion}

Tolerance design is essential in manufacturing and it plays an important role in relating performance to the manufacturing cost of a product. A good tolerance design method should be able to assign a set of tolerances for the dimensioning entities such that the maximum ranges of tolerance are obtained while satisfying the functional requirement. In this paper, a hierarchical interval constraint network is described and the techniques for 
tolerance propagation are developed. The contributions are summarized as follows:

(1) A hierarchical interval constraint network to represent the relationships among the functional requirements, attributes, and entities is developed.

(2) The techniques of forward and backward propagation for are developed. Optimization of manufacturing costs is considered in backward propagation.

\section{References}

[1] Cagan, J, and Kurfess, T. R., "Optimal Tolerance Allocation over Multiple Manufacturing Alternatives," Advances in Design Automation, DEVol. 44-2, 1992.

[2] Cleghorn, W. L., Fenton, R. G., and Fu, J. F., "Optimum Tolerancing of Planar Mechanisms Based on an Error Sensitivity Analysis," Journal of Mechanical Design, Transactions of the ASME, June, 1993

[3] Davis, E., "Constraint Propagation With Interval Labels," Artificial Intelligence, Vol. 32, p. 281-331, 1987.

[4] Dechter, R. and Pearl, J., "Tree Clustering for Constraint Networks," Artificial Intelligence, Vol. 38, p. 353-366, 1989.

[5] Fortini, E. T., Dimensioning for Interchangeable Manufacture, Industrial Press, New York, 1967.

[6] Foster, L. W., Geo-metrics II: The Application of Geometric Tolerancing Techniques, AddisonWesley, Reading, Massachusetts, 1983.

[7] Hyvonen, E., "Constraint Reasoning Based on Interval Arithmetic: the Tolerance Propagation Approach," Artificial Intelligence, Vol. 58, No. 1-3, December, p. 71-112, 1992.

[8] Krishnaswami, M., and Mayne, R. W., "Optimizing Tolerance Allocation Based on Manufacturing Cost and Quality Loss," Advances in Design Automation, vol.64, no.1, 1994.

[9] Ladkin, P. B., and Reinefeld, A., "Effective Solution of Qualitative Interval Constraint Problems," Artificial Intelligence, Vol. 57, p. 105-124, 1992.

[10] Lu, S. C. and Wilhelm, R. G., "Automating Tolerance Synthesis: a Framework and Tools," Journal of Manufacturing systems, Vol. 10, No. 4, p. 279-296, 1991.

[11] Mackworth, A. K., and Freuder, E. C., "The Complexity of Some Polynomial Network Consistency Algorithms for Constraint Satisfaction Problems," Artificial Intelligence, Vol. 25, p. 65-74, 1985.

[12] Michael, W., and Siddall, J. N., "The Optimal Tolerance Assignment with Less Than Full Acceptance," Journal of Mechanical Design, Vol. 104, October, p. 855-860, 1982.

[13] Ogot, M. M., and Gilmore, B. J., "A KinematicBased Approach for Robust Design of Mechanical Assemblies," Engineering Design \& Automation, vol.1, no.4, 1995 .
[14] Ostwald, P. F., and Huang, J., "A Method for Optimal Tolerance Selection," Journal of Engineering for Industry, Transactions of the ASME, August, 1973.

[15] Parkinson, D. B., "Assessment and Optimization of Dimensional Tolerances," Computer-Aided Design, Vol. 17, No. 4, May, p. 191-199, 1985.

[16] Söderberg, R., "Robust Design by Tolerance Allocation Considering Quality and Manufacturing Cost," Advances in Design Automation, vol.69, no. 1, 1994.

[17] Speckhart, F. H., "Calculation of Tolerance Based on a Minimum Cost Approach," Journal of Engineering for Industry, Transactions of the ASME, May, 1972.

[18] Spotts, M. F., "Allocation of Tolerances to Minimize Cost of Assembly," Journal of Engineering for Industry, Transactions of the ASME, August, 1973.

[19] Turner, J. U., "Tolerances in Computer-Aided Geometric Design," Ph.D. Dissertation, Rensselaer Polytechnic Institute, 1987.

[20] Yang, C. C., Marefat, M. M., and Ciarallo, F. W., "Tolerance Analysis and Synthesis by Interval Constraint Networks," Proceedings of IEEE International Conference on Robotics and Automation, Albuquerque, NM, April 22-25, 1997.

[21] Yang, C. C., "Active Vision Inspection: Planning, Error Analysis, and Tolerance Design," Ph.D. Dissertation, Department of Electrical and Computer Engineering, The University of Arizona, August, 1997. 